\title{
SINGULAR INTEGRALS WITH MIXED HOMOGENEITY IN PRODUCT SPACES
}

\section{LARRY CHEN AND HUNG Viet LE}

Abstract. Let $\Omega \in L\left(\log L^{+}\right)^{2}\left(S^{n-1} \times S^{m-1}\right)(n, m \geqslant 2)$ satisfy some cancellation conditions. We prove the $L^{p}$ boundedness $(1<p<\infty)$ of the singular integral

$$
T f\left(x_{1}, x_{2}\right)=\text { p. v. } \int_{\mathbb{R}^{n} \times \mathbb{R}^{m}} \frac{\Omega\left(y_{1}^{\prime}, y_{2}^{\prime}\right) h\left(\rho_{1}\left(y_{1}\right), \rho_{2}\left(y_{2}\right)\right)}{\rho_{1}^{\alpha}\left(y_{1}\right) \rho_{2}^{\beta}\left(y_{2}\right)} f\left(x_{1}-y_{1}, x_{2}-y_{2}\right) d y_{1} d y_{2},
$$

where $\rho_{1}, \rho_{2}$ are some metrics which are homogeneous with respect to certain non-isotropic dilations. We also study the above singular integral along some surfaces.

Mathematics subject classification (2010): 42B20, 42B25.

Keywords and phrases: Singular integrals, maximal functions, nonisotropic dilations, product spaces, $L^{p}$ spaces.

\section{REFERENCES}

[1] Ahmad Al-Salman, Maximal functions along surfaces on product domains, Anal. Math., 34, 3 (2008), 163-175.

[2] Ahmad Al-Salman And Yibiao Pan, Singular integrals with rough kernels in $\operatorname{Llog} L\left(S^{n-1}\right), \mathrm{J}$. London Math. Soc. (2), 66 (2002), 153-174.

[3] Ahmad Al-Salman, H. Al-QASSEM And Yibiao PAn, Singular integrals on product domains, Indiana Univ. Math. J., 55, 1 (2006), 369-387.

[4] YANPIng CHEn, Yong Ding And Dashan FAn, A parabolic singular integral operator with rough kernel, submitted.

[5] J. DuoAndikoetxea, Multiple singular integrals and maximal functions along hypersurfaces, Annales de l'institut Fourier, 36, 4 (1986), 185-206.

[6] DASHAN FAN AND YiBIAO PAN, Singular integral operators with rough kernels supported by subvarieties, Amer. J. Math., 119, 4 (1997), 799-839.

[7] JAVIER DUOANDiKoetXeA, Jos É L. Rubio DE Francia, Maximal and singular integral operators via Fourier transform estimates, Invent. Math., 84, 3 (1986), 541-561.

[8] Robert FEFFERMAn, Singular integrals on product spaces, Advances in Mathematics, 45 (1982), 117-143.

[9] E. B. FABES AND N. M. RIVIÈRE, Singular integrals with mixed homogeneity, Studia Mathematica, 27 (1966), 19-38.

[10] G. B. Folland And E. M. Stein, Hardy spaces on homogeneous groups, Mathematical Notes 28 , Princeton University Press and University of Tokyo Press, 1982.

[11] H. V. LE, Maximal function along surfaces in product spaces, J. Math. Anal. Appl., 316 (2006), $422-432$.

[12] A. Nagel, N. M. Rivière And S. Wainger, On Hilbert transforms along curves, II, Amer. J. Math., 98 (1976), 395-403.

[13] Elias M. Stein, Harmonic Analysis: Real-Variable Methods, Orthogonality, and Oscillatory Integrals, Princeton University Press, 1993.

[14] E. M. Stein, S. Wainger, Problems in Harmonic Analysis related to curvature, Bull. Amer. Math. Soc., 84 (1978), 1239-1295. 